\title{
DETERMINAÇÃO DE TEORES ÓTIMOS DE NUTRIENTES EM SOJA PELOS MÉTODOS CHANCE MATEMÁTICA, SISTEMA INTEGRADO DE DIAGNOSE E RECOMENDAÇÃO E DIAGNOSE DA COMPOSIÇÃO NUTRICIONAL ${ }^{(1)}$
}

\author{
Eliane Oshiro Mocelin Urano ${ }^{(2)}$, Carlos Hissao Kurihara ${ }^{(3)}$, Shizuo \\ Maeda $^{(3)}$, Antonio Carlos Tadeu Vitorino ${ }^{(4)}$, Manoel Carlos \\ Gonçalves $^{(4)}$ \& Marlene Estevão Marchetti ${ }^{(4)}$
}

\begin{abstract}
RESUMO
Diversos estudos têm demonstrado o uso promissor de métodos de diagnose nutricional para definição de teores ótimos e níveis críticos de nutrientes em tecidos vegetais. Nesse sentido, o objetivo deste trabalho foi comparar os teores ótimos de nutrientes para soja, estimados por meio dos métodos Chance Matemática (ChM), Sistema Integrado de Diagnose e Recomendação (DRIS) e Diagnose da Composição Nutricional (CND), a partir de dados provenientes de monitoramento nutricional de 111 lavouras comerciais de soja, da região sul do Estado de Mato Grosso do Sul. Os teores ótimos de nutrientes estimados pelos métodos DRIS e CND foram idênticos ao teor médio observado na população de referência. Para o método $\mathrm{ChM}$, exceto para os nutrientes $\mathrm{Cu}, \mathrm{Fe}, \mathrm{Mn}$ e $\mathrm{Zn}$, os teores ótimos estimados também foram idênticos ou muito próximos ao teor médio na população de referência. Os métodos ChM, DRIS e CND mostraram-se promissores na calibração de teores ótimos para a cultura da soja a partir de dados provenientes de monitoramentos nutricionais de lavouras comerciais.
\end{abstract}

Termos de indexação: diagnose foliar, estado nutricional, Glycine max.

(1) Parte da Tese de Mestrado do primeiro autor apresentada ao Departamento de Ciências Agrárias da Universidade Federal de Mato Grosso do Sul - UFMS. Recebido para publicação em maio de 2005 e aprovado em janeiro de 2007.

(2) Engenheiro-Agrônomo da Sementes Barreirão Ltda. Rodovia BR 376, km 09, Caixa Postal 1005, CEP $79830-970$ Dourados (MS). E-mail: elimocelin@terra.com.br

(3) Pesquisador da Embrapa Agropecuária Oeste, Rodovia BR 163, km 253 Caixa Postal 661, CEP 79804-970 Dourados (MS). E-mails: kurihara@cpao.embrapa.br; maeda@cpao.embrapa.br

(4) Professor do Departamento de Ciências Agrárias da Universidade Federal de Mato Grosso do Sul - UFMS. Caixa Postal 533, CEP 74804-970 Dourados (MS). E-mails: vitorino@ceud.ufms.br; mancgonc@ceud.ufms.br; emarche@ceud.ufms.br 


\title{
SUMMARY: DETERMINATION OF OPTIMAL NUTRIENT CONTENTS FOR SOYBEANBYTHE MATHEMATICAL CHANCE, DIAGNOSIS AND RECOMMENDATION INTEGRATED SYSTEM AND COMPOSITIONAL NUTRIENT DIAGNOSIS METHODS
}

\begin{abstract}
Several studies pointed out the promising use of nutritional diagnosis methods for the determination of optimum nutrient contents and critical levels in plant tissues. Thus, the objective of this study was to compare the optimum nutrient content for soybean, estimated by the Mathematical Chance (ChM), Diagnosis and Recommendation Integrated System (DRIS) and Compositional Nutrient Diagnosis (CND) methods. The optimum nutrient contents estimated by DRIS and CND where identical to the mean content in the reference population. Apart from the nutrients $\mathrm{Cu}, \mathrm{Fe}, \mathrm{Mn}$ and $\mathrm{Zn}$, the optimum contents were also equal or in close proximity to the mean nutrient contents of the reference population by the ChM method. The ChM, DRIS and CND methods proved promising for the calibration of optimal nutrient contents for soybean based on data obtained from nutrient monitoring of commercial fields.
\end{abstract}

Index terms: foliar diagnosis, nutritional status, Glycine max.

\section{INTRODUÇÃO}

Níveis críticos para os teores de nutrientes em tecidos vegetais têm sido definidos por meio de abordagem intervencionista, em que a variabilidade da produção é explicada por variações no suprimento ou na disponibilidade do nutriente em análise, sendo os demais fatores de produção, nutricionais ou não, mantidos em níveis não-limitantes.

Quanto maior for a similaridade entre as condições edafoclimáticas e vegetais da lavoura que se deseja diagnosticar e as condições em que foram estabelecidos os níveis críticos, maior será a confiabilidade do diagnóstico nutricional. Isso implica a necessidade de implementação de ensaios de calibração em vários locais e ao longo do tempo, a fim de assegurar que diferenças em características de solo, clima e potencial produtivo de espécies vegetais estejam sendo consideradas para o estabelecimento de níveis críticos.

Entretanto, Kurihara (2004) relata que a interpretação de teores de nutrientes em folhas de soja no Estado de Mato Grosso do Sul (Embrapa, 2003) baseia-se, com pequenas alterações, em resultados de pesquisa obtidos no final da década de 1980 em seis regiões norte-americanas (Sfredo et al., 1986).

O método da Chance Matemática objetiva determinar a faixa de valores de um dado fator, interno ou externo à planta, em que se espera obter a máxima produtividade, possibilitando a determinação de um nível crítico, nível ótimo e faixa de suficiência a partir de dados provenientes de monitoramentos nutricionais (Wadt, 1996), tendo sido empregado nas culturas de eucalipto (Wadt et al., 1998a,b) e soja (Kurihara, 2004).

Em amostras de tecidos vegetais, em que as relações duais entre teores de nutrientes apresentem valores similares aos estabelecidos como normas, o índice DRIS para cada nutriente se aproxima de zero
(Dara et al., 1992). Partindo desse princípio, modelos estatísticos têm sido ajustados para descrever o relacionamento entre índices DRIS (Sistema Integrado de Diagnose e Recomendação) e teores de nutrientes no tecido vegetal em eucalipto (Wadt et al., 1998b), café (Reis Júnior et al., 2002), cana-de-açúcar (Reis Júnior \& Monnerat, 2003) e soja (Kurihara, 2004), com a finalidade de aprimorar a interpretação de teores de nutrientes em tecidos vegetais.

Apesar de um valor de índice DRIS ou CND (Diagnose da Composição Nutricional) igual a zero ser considerado ótimo e todos os outros valores, sub ou supra-ótimos, erros experimentais, como os relacionados às determinações analíticas e pesagens, assim como limitações devidas ao tamanho da amostra, causam redução da confiabilidade da estimativa pontual do estado ótimo nutricional (Hartz et al., 1998). Em razão disso, esses autores sugerem a utilização de uma faixa ótima para avaliar os índices nutricionais. Kurihara (2004) considera equilibrado o índice de um nutriente cujo valor esteja localizado no intervalo de $2 / 3$ desvios-padrão em relação ao índice DRIS ou CND igual a zero, obtido a partir da subpopulação de alta produtividade.

Nesse sentido, o objetivo deste trabalho foi comparar os teores ótimos de nutrientes para soja, estimados por meio dos métodos Chance Matemática, DRIS e CND, a partir de dados provenientes de monitoramento nutricional de 111 lavouras comerciais de soja, amostradas na região sul do Estado de Mato Grosso do Sul.

\section{MATERIAL E MÉTODOS}

Foram coletadas amostras de tecido foliar em 27 lavouras comerciais de soja cultivadas no sistema 
plantio direto, situadas na região sul do Estado de Mato Grosso do Sul, durante o ano agrícola de 2001/ 2002. Em cada lavoura, no estádio de desenvolvimento R2 (Costa \& Marchezan, 1982), constituiu uma amostra composta o terceiro trifólio (incluindo o pecíolo) a partir do ápice da planta, coletado em 30 plantas aleatoriamente escolhidas. No estádio R8 (Costa \& Marchezan, 1982), determinou-se a produtividade de grãos a partir da amostragem de três linhas de cultivo, com $2 \mathrm{~m}$ de comprimento cada e espaçamento de 0,45 m entre linhas, em quatro locais aleatoriamente escolhidos. Constituíram o banco de dados, além das 27 já mencionadas, 84 amostras coletadas por Maeda (2002) em lavouras comerciais de soja cultivadas no sistema plantio direto, situadas na região sul do Estado de Mato Grosso do Sul, durante o ano agrícola de 2000/2001, no estádio de desenvolvimento $\mathrm{R} 1$. Os nutrientes avaliados em ambos os conjuntos de amostras foram N, P, K, Ca, $\mathrm{Mg}, \mathrm{S}, \mathrm{B}, \mathrm{Cu}, \mathrm{Fe}, \mathrm{Mn}$ e $\mathrm{Zn}$, de acordo com os procedimentos indicados por Malavolta et al. (1997).

Do conjunto de amostras constituintes do banco de dados, foram definidas como subpopulação de alta produtividade aquelas amostras cujo rendimento de grãos foi superior à média + 0,5 desvio-padrão, ou seja, $4.400 \mathrm{~kg} \mathrm{ha}^{-1}$.

\section{Chance Matemática (ChM)}

Para cada nutriente em estudo, os teores foliares foram classificados em ordem crescente e distribuídos em um número de classes definido como sendo a raiz quadrada do número de observações. Dessa forma, quanto maior for o número de observações, maior será o número de classes, aumentando a sensibilidade do método para identificar diferenças de produtividade considerando os teores de nutrientes no tecido foliar. Os intervalos de valores de cada classe foram então determinados, dividindo-se a amplitude dos teores do nutriente em questão pelo número de classes estabelecido, de acordo com Wadt (1996). Em cada classe, calculou-se a chance matemática $\left(\mathrm{ChM}_{\mathrm{i}}\right)$ conforme a equação:

$$
\operatorname{ChM}_{\mathrm{i}}=\left(\operatorname{ChM}\left(\mathrm{A}_{\mathrm{i}} / \mathrm{A}\right) \times \operatorname{ChM}\left(\mathrm{A}_{\mathrm{i}} / \mathrm{C}_{\mathrm{j}}\right)\right)^{0,5}
$$

em que $\operatorname{ChM}\left(\mathrm{A}_{\mathrm{i}} / \mathrm{A}\right)=\mathrm{P}\left(\mathrm{A}_{\mathrm{i}} / \mathrm{A}\right) \times \mathrm{PROD}_{\mathrm{i}} ; \mathrm{P}\left(\mathrm{A}_{\mathrm{i}} / \mathrm{A}\right)=$ freqüência de lavouras de alta produtividade na classe $i$, em relação ao total geral de talhões de alta produtividade; $\mathrm{PROD}_{\mathrm{i}}=$ produtividade média dos talhões de alta produtividade na classe i $\left(\mathrm{kg} \mathrm{ha}^{-1}\right)$; $\operatorname{ChM}\left(\mathrm{A}_{\mathrm{i}} / \mathrm{C}_{\mathrm{i}}\right)=\mathrm{P}\left(\mathrm{A}_{\mathrm{i}} / \mathrm{C}_{\mathrm{i}}\right) \times \mathrm{PROD}_{\mathrm{i}}$; e $\mathrm{P}\left(\mathrm{A}_{\mathrm{i}} / \mathrm{C}_{\mathrm{i}}\right)=$ freqüência de talhões de alta produtividade na classe $\mathrm{i}$, em relação ao total geral de talhões na classe $i$.

Para cada nutriente, a faixa ótima consistiu da(s) classe(s) de valores que apresentou(ram) maior(es) valor(es) de chance matemática, sendo o seu limite inferior considerado o nível crítico e a sua mediana o valor ótimo do fator de produção.

\section{Sistema Integrado de Diagnose e Recomendação (DRIS)}

A média aritmética e o desvio-padrão de todos os quocientes entre as concentrações de nutrientes $(\mathrm{A} / \mathrm{B}$, $\mathrm{A} / \mathrm{C}, \mathrm{A} / \mathrm{N}, \ldots, \mathrm{B} / \mathrm{A}, \mathrm{C} / \mathrm{A}, \mathrm{N} / \mathrm{A})$, transformadas por função logarítmica neperiana, na população de alta produtividade, constituíram as normas ou valorespadrão. Em função destas, calcularam-se as variáveis normais reduzidas dos logaritmos neperianos dos quocientes na subpopulação de alta produtividade. $\mathrm{O}$ desvio do logaritmo neperiano de um quociente em uma amostra $(\mathrm{A} / \mathrm{B})$, em relação ao valor médio do logaritmo neperiano do mesmo quociente na população de alta produtividade $(\mathrm{a} / \mathrm{b})$, foi determinado em unidades de desvio-padrão (s), utilizando-se um fator de ajuste $\mathrm{c}=1$, conforme sugerido por Wadt et al. (1998a):

$$
\mathrm{Z}(\mathrm{A} / \mathrm{B})=[(\mathrm{A} / \mathrm{B})-(\mathrm{a} / \mathrm{b})] / \mathrm{s}
$$

Os índices DRIS (Jones, 1981), modificado por Jones et al. (1998a) para cada nutriente consistiram da média aritmética dos quocientes transformados em variáveis normais reduzidas, de acordo com Alvarez \& Leite (1999):

$$
\begin{gathered}
\mathrm{I}_{\mathrm{A}}=[\mathrm{Z}(\mathrm{A} / \mathrm{B})+\mathrm{Z}(\mathrm{A} / \mathrm{C})+\mathrm{Z}(\mathrm{A} / \mathrm{N})+\ldots \\
-\mathrm{Z}(\mathrm{B} / \mathrm{A})-\mathrm{Z}(\mathrm{C} / \mathrm{A})-\mathrm{Z}(\mathrm{N} / \mathrm{A})] / 2(\mathrm{n}-1)
\end{gathered}
$$

Como valores nulos dos índices DRIS caracterizam uma condição de equilíbrio nutricional, uma vez que os valores dos índices refletem os desvios padronizados em relação aos valores de referência, estimaram-se os teores ótimos de nutrientes por meio do ajuste de modelos estatísticos ao relacionamento entre índices DRIS e teores foliares de nutrientes, na subpopulação de alta produtividade. Dessa forma, os teores de nutrientes associados a valores de índices DRIS nulos corresponderam aos teores ótimos estimados em estudos de Wadt et al. (1998b), Silva (2001), Reis Júnior et al. (2002), Reis Júnior \& Monnerat (2003) e Kurihara (2004). Os limites inferior e superior da faixa ótima foram considerados como as concentrações dos nutrientes estimadas quando os índices DRIS corresponderam ao seu valor nulo $\pm 2 / 3$ desvio-padrão (Faixas de Beaufils), respectivamente, conforme Kurihara (2004), que considerou equilibrados os valores dos índices DRIS situados dentro do intervalo entre $-102 / 3$ s e $102 / 3$ s, em virtude de ter utilizado um fator de ajuste (c) igual a 10, com o objetivo de aproximar os valores dos índices a números inteiros.

\section{Diagnose da Composição Nutricional (CND)}

As normas CND foram constituídas da média aritmética e do desvio-padrão das variáveis multinutrientes na população de alta produtividade, de acordo com Khiari et al. (2001a,b).

As variáveis multinutrientes $\left(V_{\mathrm{A}}\right)$ consistiram dos valores logaritmizados dos quocientes entre a concentração de cada nutriente (A) e a média geométrica dos teores dos constituintes da massa seca $(\mathrm{G})$ : 


$$
V_{\mathrm{A}}=\ln (\mathrm{A} / \mathrm{G})
$$

Os índices CND foram calculados pela diferença entre as variáveis multinutrientes no talhão avaliado $\left(V_{\mathrm{A}}\right)$ e na média da população de referência $\left(V_{\mathrm{A}}^{*}\right)$, dividido pelo desvio-padrão desta variável na população de referência $\left(\mathrm{s}_{\mathrm{A}}{ }^{*}\right)$ :

$$
\mathrm{I}_{\mathrm{A}}=\left(V_{\mathrm{A}}-V_{\mathrm{A}}^{*}\right) / \mathrm{s}_{\mathrm{A}} *
$$

Os valores dos teores ótimos, limite inferior e superior da faixa ótima de concentração de nutrientes pelo método CND, foram determinados de modo análogo ao utilizado pelo método DRIS.

\section{RESULTADOS E DISCUSSÃO}

Apesar de o banco de dados ser constituído de amostras representativas de diferentes estádios de desenvolvimento, de acordo com Ritchie et al. (1997), em uma dada lavoura de soja, nem todas as plantas apresentam-se simultaneamente no mesmo estádio de desenvolvimento e cada estádio específico é determinado quando $50 \%$ ou mais das plantas na lavoura apresentam-se neste estádio. Considerou-se, portanto, que, sendo os estádios R1 e R2 sucessivos, podem ocorrer simultaneamente em uma mesma lavoura, e, apesar de representar uma fonte de variabilidade, esta foi empregada, possibilitando a utilização de maior número de amostras para formação do banco de dados. No tocante à variabilidade ocasionada pela utilização de amostras coletadas em diferentes safras, considerou-se que, em se tratando de amostras coletadas em uma mesma região geográfica, a variabilidade ocasionada por fatores que variem ao longo do tempo, como condições climáticas, pode aumentar a representatividade de um banco de dados de caráter regional.

\section{Chance Matemática (ChM)}

Para o N, os maiores valores de Chance Matemática ocorreram nas classes de concentração de número $4 \mathrm{a}$ 7 (Quadro 1). Isso se deve, principalmente, à maior proporção de talhões de alta produtividade nestas classes, em relação ao número total de talhões de alta produtividade $\left(\mathrm{P}\left(\mathrm{A}_{\mathrm{i}} / \mathrm{A}\right)\right)$ e, em menor extensão, à maior proporção de talhões de alta produtividade em relação ao número total de talhões em cada classe $\left(\mathrm{P}\left(\mathrm{A}_{\mathrm{i}} / \mathrm{C}_{\mathrm{i}}\right)\right.$.

Pode-se ainda verificar alta proporção de talhões de alta produtividade em relação ao número total de talhões nas classes 2 e 10 (Quadro 1). Contudo, como nestas duas classes o número de amostras era pequeno, a participação das amostras de alta produtividade presentes nestas classes, em relação ao total de talhões da população de referência $\left(\mathrm{P}\left(\mathrm{A}_{\mathrm{i}} / \mathrm{A}\right)\right)$, era reduzida, o que resultou em menores valores de Chance Matemática (Quadro 1).
De maneira semelhante, foram determinados os valores de Chance Matemática para as classes de teores de outros nutrientes avaliados (Quadro 1), sendo que semelhantemente ao $\mathrm{N}$, para o $\mathrm{Se}$ o $\mathrm{Zn}$, os maiores valores de Chance Matemática ocorreram nas classes que apresentaram, de maneira geral, a maior proporção de talhões de alta produtividade, em relação ao número total de talhões de alta produtividade $\left(\mathrm{P}\left(\mathrm{A}_{\mathrm{i}}\right)\right.$ A). Diferentemente, para o Ca e o B, as classes com maiores valores de Chance Matemática foram encontradas entre aquelas que apresentaram, de modo geral, maior proporção de talhões de alta produtividade em relação ao número total de talhões em cada classe $\left(\mathrm{P}\left(\mathrm{A}_{\mathrm{i}} / \mathrm{C}_{\mathrm{i}}\right)\right.$. Para outros nutrientes, como o $\mathrm{P}$, o $\mathrm{K}$, o $\mathrm{Mg}$, o $\mathrm{Cu}$, o Fe e o $\mathrm{Mn}$, tanto a maior proporção de talhões de alta produtividade em relação ao número total de talhões de alta produtividade $\left(\mathrm{P}\left(\mathrm{A}_{\mathrm{i}} / \mathrm{A}\right)\right.$ quanto a maior proporção de talhões de alta produtividade em relação ao número total de talhões em cada classe $\left(\mathrm{P}\left(\mathrm{A}_{\mathrm{i}} / \mathrm{C}_{\mathrm{j}}\right)\right.$ foram determinantes para definição das classes de teores com maiores valores de Chance Matemática, ou seja, os limites inferior e superior da faixa ótima de nutrientes (Quadro 3), à qual está relacionada a maior possibilidade de obtenção de altas produtividades.

\section{Sistema Integrado de Diagnose e Recomendação (DRIS)}

Todos os modelos estatísticos ajustados aos relacionamentos entre os índices DRIS e os teores de nutrientes em trifólios de soja apresentaram significância estatística $(p<0,001)$, com coeficientes de determinação variando de 0,53 para $P$ até 0,86 para Zn (Quadro 2).

A tentativa de ajustar modelos de regressão a esses relacionamentos resultou, com freqüência, na obtenção de coeficientes de regressão associados às variáveis de segundo e terceiro graus muito próximos de zero, indicando não serem esses modelos adequados.

De acordo com Kurihara (2004), desvios de simetria na distribuição das relações duais podem ocasionar curvaturas na dispersão de pontos, quando se relacionam índices DRIS em função de teor foliar ou vice-versa. Em todos os nutrientes avaliados, o teor ótimo estimado (TOE) pelo relacionamento dos índices DRIS com teores foliares correspondeu, assim como para Kurihara (2004), ao teor médio na subpopulação de referência (Quadro 3).

\section{Diagnose da Composição Nutricional (CND)}

$\mathrm{O}$ ajuste de modelos estatísticos lineares ao relacionamento entre os índices CND e os teores de nutrientes em folhas de soja resultou na obtenção de coeficientes de determinação muito próximos aos obtidos por meio de ajuste de modelos lineares ao relacionamento entre índices DRIS e teores de nutrientes (Quadro 2). O teor ótimo estimado (TOE) também correspondeu ao teor médio do nutriente na 
Quadro 1. Valores de chance matemática $(\mathrm{ChM})^{(1)}$ estabelecidos para diferentes classes da distribuição de teores de nutrientes em amostras de terceiro trifólio, coletadas na região sul do Estado de Mato Grosso do Sul, nos anos agrícolas de 2000/2001 e 2001/2002

\begin{tabular}{|c|c|c|c|c|c|c|}
\hline Classe $^{(1)}$ & $\mathbf{L} \mathbf{I}_{\mathbf{i}}{ }^{(2)}$ & $\mathbf{L S}_{\mathbf{i}^{(3)}}$ & (PRODi) $^{(4)}$ & $\mathbf{P}\left(\mathbf{A}_{\mathrm{i}} / \mathbf{A}\right)^{(5)}$ & $\mathbf{P}\left(\mathrm{A}_{\mathrm{i}} / \mathrm{C}_{\mathrm{i}}\right)^{(6)}$ & $\operatorname{ChM}_{i}{ }^{(7)}$ \\
\hline & g l & - & $\mathrm{kg} \mathrm{ha-1}$ & & & $\mathrm{kg} \mathrm{ha} \mathrm{h}^{-1}$ \\
\hline & & & Nitrogênio & & & \\
\hline 1 & 27,0 & 30,0 & 4948 & 0,026 & 0,200 & 354 \\
\hline 2 & 30,1 & 32,9 & 4582 & 0,103 & 0,333 & 847 \\
\hline 3 & 33,0 & 35,9 & 4874 & 0,103 & 0,200 & 698 \\
\hline 4 & 36,0 & 38,9 & 4709 & 0,256 & 0,455 & 1.608 \\
\hline 5 & 39,0 & 41,9 & 5032 & 0,128 & 0,313 & 1.007 \\
\hline 6 & 42,0 & 44,8 & 4614 & 0,154 & 0,500 & 1.280 \\
\hline 7 & 44,9 & 47,8 & 4561 & 0,128 & 0,556 & 1.217 \\
\hline 8 & 47,9 & 50,8 & 4410 & 0,026 & 0,125 & 250 \\
\hline 9 & 50,9 & 53,7 & 4445 & 0,026 & 0,250 & 356 \\
\hline \multirow[t]{2}{*}{10} & 53,8 & 56,7 & 4485 & 0,051 & 0,667 & 829 \\
\hline & & & Fósforo & & & \\
\hline 1 & 1,3 & 1,6 & - & - & - & - \\
\hline 2 & 1,7 & 1,9 & - & - & - & - \\
\hline 3 & 2,0 & 2,2 & 4622 & 0,077 & 0,333 & 740 \\
\hline 4 & 2,3 & 2,5 & 4989 & 0,103 & 0,286 & 854 \\
\hline 5 & 2,6 & 2,8 & 4613 & 0,205 & 0,444 & 1.393 \\
\hline 6 & 2,9 & 3,0 & 4662 & 0,231 & 0,310 & 1.248 \\
\hline 7 & 3,1 & 3,3 & 4678 & 0,256 & 0,556 & 1.766 \\
\hline 8 & 3,4 & 3,6 & 4775 & 0,128 & 0,556 & 1.274 \\
\hline 9 & 3,7 & 3,9 & - & - & - & - \\
\hline \multirow[t]{2}{*}{10} & 4,0 & 4,2 & - & - & - & - \\
\hline & & & Potássio & & & \\
\hline 1 & 12,0 & 14,3 & 4549 & 0,026 & 0,200 & 326 \\
\hline 2 & 14,4 & 16,5 & 5210 & 0,026 & 0,143 & 315 \\
\hline 3 & 16,6 & 18,8 & 4777 & 0,128 & 0,417 & 1.104 \\
\hline 4 & 18,9 & 21,0 & 4925 & 0,026 & 0,077 & 219 \\
\hline 5 & 21,1 & 23,3 & 4562 & 0,154 & 0,400 & 1.132 \\
\hline 6 & 23,4 & 25,5 & 4705 & 0,462 & 0,474 & 2.200 \\
\hline 7 & 25,6 & 27,8 & 4608 & 0,103 & 0,308 & 819 \\
\hline 8 & 27,9 & 30,0 & 4759 & 0,077 & 0,429 & 864 \\
\hline 9 & 30,1 & 32,3 & - & - & - & - \\
\hline \multirow[t]{2}{*}{10} & 32,4 & 34,5 & - & - & - & - \\
\hline & & & Cálcio & & & \\
\hline 1 & 4,4 & 5,8 & - & - & - & - \\
\hline 2 & 5,9 & 7,1 & - & - & - & - \\
\hline 3 & 7,2 & 8,5 & 4795 & 0,103 & 0,333 & 887 \\
\hline 4 & 8,6 & 9,8 & 4809 & 0,205 & 0,333 & 1.258 \\
\hline 5 & 9,9 & 11,2 & 4570 & 0,256 & 0,313 & 1.294 \\
\hline 6 & 11,3 & 12,6 & 4710 & 0,231 & 0,391 & 1.415 \\
\hline 7 & 12,7 & 13,9 & - & - & - & - \\
\hline 8 & 14,0 & 15,3 & 4749 & 0,051 & 0,667 & 878 \\
\hline 9 & 15,4 & 16,6 & 4645 & 0,103 & 0,800 & 1.331 \\
\hline \multirow[t]{2}{*}{10} & 16,7 & 18,0 & 4761 & 0,051 & 0,500 & 762 \\
\hline & & & Magnésio & & & \\
\hline 1 & 1,7 & 2,1 & 4611 & 0,021 & 0,450 & 1.486 \\
\hline 2 & 2,2 & 2,6 & 4879 & 0,014 & 0,545 & 1.413 \\
\hline 3 & 2,7 & 3,0 & 4699 & 0,007 & 0,429 & 853 \\
\hline 4 & 3,1 & 3,4 & 4711 & 0,014 & 0,353 & 1.098 \\
\hline 5 & 3,5 & 3,9 & 4421 & 0,005 & 0,182 & 427 \\
\hline 6 & 4,0 & 4,3 & 4702 & 0,023 & 0,370 & 1.449 \\
\hline 7 & 4,4 & 4,7 & 4763 & 0,002 & 0,111 & 254 \\
\hline 8 & 4,8 & 5,1 & 4789 & 0,005 & 0,250 & 542 \\
\hline 9 & 5,2 & 5,6 & - & - & - & - \\
\hline \multirow[t]{2}{*}{10} & 5,7 & 6,0 & - & - & - & - \\
\hline & & & Enxofre & & & \\
\hline 1 & 1,3 & 1,6 & 4648 & 0,077 & 0,600 & 999 \\
\hline 2 & 1,7 & 1,9 & 4601 & 0,205 & 0,615 & 1.635 \\
\hline 3 & 2,0 & 2,2 & 4775 & 0,256 & 0,303 & 1.331 \\
\hline 4 & 2,3 & 2,5 & 4706 & 0,231 & 0,391 & 1.414 \\
\hline 5 & 2,6 & 2,8 & 4766 & 0,103 & 0,182 & 651 \\
\hline 6 & 2,9 & 3,1 & 4420 & 0,026 & 0,125 & 250 \\
\hline 7 & 3,2 & 3,4 & 4769 & 0,051 & 0,500 & 764 \\
\hline 8 & 3,5 & 3,7 & 4948 & 0,026 & 0,500 & 560 \\
\hline 9 & 3,8 & 4,0 & - & - & - & - \\
\hline 10 & 4,1 & 4,3 & 4513 & 0,026 & 1,000 & 723 \\
\hline
\end{tabular}


Quadro 1. Continuação

\begin{tabular}{|c|c|c|c|c|c|c|}
\hline Classe $^{(1)}$ & $\mathbf{L} \mathbf{I}_{\mathbf{i}}{ }^{(2)}$ & $\mathbf{L} \mathbf{S}_{\mathbf{i}^{(3)}}$ & (PRODi) $^{(4)}$ & $\mathbf{P}\left(\mathbf{A}_{\mathrm{i}} / \mathrm{A}\right)^{(5)}$ & $\mathbf{P}\left(\mathbf{A}_{\mathrm{i}} / \mathbf{C}_{\mathrm{i}}\right)^{(6)}$ & $\operatorname{ChM}_{i^{(7)}}$ \\
\hline & \multicolumn{2}{|c|}{$\mathrm{mg} \mathrm{kg}^{-1}$} & $\mathrm{~kg} h \mathrm{a}^{-1}$ & & & $\mathrm{~kg} \mathrm{ha-1}$ \\
\hline & & & Boro & & & \\
\hline 1 & 23,8 & 27,5 & 4753 & 0,026 & 0,250 & 381 \\
\hline 2 & 27,6 & 31,2 & 4759 & 0,026 & 1,000 & 762 \\
\hline 3 & 31,3 & 35,0 & 4935 & 0,077 & 0,333 & 790 \\
\hline 4 & 35,1 & 38,7 & 4819 & 0,154 & 0,300 & 1.035 \\
\hline 5 & 38,8 & 42,4 & 4585 & 0,282 & 0,393 & 1.526 \\
\hline 6 & 42,5 & 46,1 & 4815 & 0,205 & 0,471 & 1.496 \\
\hline 7 & 46,2 & 49,8 & 4410 & 0,026 & 0,083 & 204 \\
\hline 8 & 49,9 & 53,6 & 4827 & 0,077 & 0,300 & 733 \\
\hline 9 & 53,7 & 57,3 & 4430 & 0,077 & 0,750 & 1.064 \\
\hline \multirow[t]{2}{*}{10} & 57,4 & 61,0 & 4484 & 0,051 & 0,333 & 586 \\
\hline & & & Cobre & & & \\
\hline 1 & 2,6 & 4,1 & 4785 & 0,026 & 1,000 & 766 \\
\hline 2 & 4,2 & 5,5 & 4576 & 0,026 & 0,071 & 196 \\
\hline 3 & 5,6 & 7,0 & 4652 & 0,128 & 0,500 & 1.178 \\
\hline 4 & 7,1 & 8,4 & 4759 & 0,308 & 0,500 & 1.867 \\
\hline 5 & 8,5 & 9,9 & 4708 & 0,179 & 0,583 & 1.523 \\
\hline 6 & 10,0 & 11,4 & 4651 & 0,077 & 0,375 & 790 \\
\hline 7 & 11,5 & 12,8 & 4545 & 0,103 & 0,174 & 607 \\
\hline 8 & 12,9 & 14,3 & 4937 & 0,051 & 0,182 & 477 \\
\hline 9 & 14,4 & 15,7 & 4633 & 0,051 & 0,333 & 606 \\
\hline \multirow[t]{2}{*}{10} & 15,8 & 17,2 & 4689 & 0,051 & 1,000 & 1.062 \\
\hline & & & Ferro & & & \\
\hline 1 & 30,9 & 51,9 & 5430 & 0,026 & 0,071 & 232 \\
\hline 2 & 52,0 & 72,9 & 4674 & 0,205 & 0,286 & 1.131 \\
\hline 3 & 73,0 & 93,9 & 4611 & 0,333 & 0,382 & 1.646 \\
\hline 4 & 94,0 & 114,9 & 4807 & 0,308 & 0,480 & 1.848 \\
\hline 5 & 115,0 & 135,9 & 4615 & 0,103 & 0,667 & 1.207 \\
\hline 6 & 136,0 & 156,8 & - & - & - & - \\
\hline 7 & 156,9 & 177,8 & 4421 & 0,026 & 1,000 & 708 \\
\hline 8 & 177,9 & 198,8 & - & - & - & - \\
\hline 9 & 198,9 & 219,8 & - & - & - & - \\
\hline \multirow[t]{2}{*}{10} & 219,9 & 240,8 & - & - & - & - \\
\hline & & & Manganês & & & \\
\hline 1 & 19,0 & 34,1 & 4568 & 0,051 & 0,200 & 463 \\
\hline 2 & 34,2 & 49,2 & 4693 & 0,385 & 0,577 & 2.210 \\
\hline 3 & 49,3 & 64,3 & 4793 & 0,231 & 0,321 & 1.305 \\
\hline 4 & 64,4 & 79,4 & 4633 & 0,205 & 0,381 & 1.295 \\
\hline 5 & 79,5 & 94,5 & 4752 & 0,077 & 0,250 & 659 \\
\hline 6 & 94,6 & 109,6 & 4925 & 0,026 & 0,167 & 322 \\
\hline 7 & 109,7 & 124,7 & 4429 & 0,026 & 0,333 & 410 \\
\hline 8 & 124,8 & 139,8 & - & - & - & - \\
\hline 9 & 139,9 & 154,9 & - & - & - & - \\
\hline \multirow[t]{2}{*}{10} & 155,0 & 170,0 & - & - & - & - \\
\hline & & & Zinco & & & \\
\hline 1 & 21,0 & 36,3 & 4522 & 0,175 & 0,259 & 963 \\
\hline 2 & 36,4 & 51,6 & 4737 & 0,250 & 0,250 & 1.184 \\
\hline 3 & 51,7 & 66,9 & 4797 & 0,225 & 0,429 & 1.490 \\
\hline 4 & 67,0 & 82,2 & 4729 & 0,225 & 0,750 & 1.943 \\
\hline 5 & 82,3 & 97,5 & 4753 & 0,050 & 0,333 & 614 \\
\hline 6 & 97,6 & 112,8 & 4421 & 0,025 & 0,500 & 494 \\
\hline 7 & 112,9 & 128,1 & 4652 & 0,025 & 0,500 & 520 \\
\hline 8 & 128,2 & 143,4 & - & - & - & - \\
\hline 9 & 143,5 & 158,7 & - & - & - & - \\
\hline 10 & 158,8 & 174,0 & - & - & - & - \\
\hline
\end{tabular}

(1) Wadt (1996). ${ }^{(2)}$ Limite inferior da classe "i”. ${ }^{(3)}$ Limite superior da classe "i”. (4) Produtividade média dos talhões de alta produtividade na classe "i". ${ }^{(5)}$ Freqüência de talhões de alta produtividade na classe "i" em relação ao total de talhões de alta produtividade. ${ }^{(6)}$ Freqüência de talhões de alta produtividade na classe "i" em relação ao total de talhões na classe "i". ${ }^{(7)}$ Chance matemática na classe "i". 
Quadro 2. Modelos estatísticos dos relacionamentos entre teores de nutrientes e índices DRIS ${ }^{(1)}$ e CND $^{(2)}$, em amostras de soja coletadas na região sul do Estado de Mato Grosso do Sul, nos anos agrícolas de 2000/ 2001 e 2001/2002, em subpopulação de alta produtividade ${ }^{(3)}$

\begin{tabular}{|c|c|c|c|}
\hline Nutriente & Método & Modelo estatístico & $\mathbf{R}^{2}$ \\
\hline \multirow[t]{2}{*}{$\mathrm{N}$} & DRIS & $\hat{\mathrm{N}}=40,595+7,856^{* * *} \mathrm{I}_{\mathrm{N}}$ & 0,73 \\
\hline & CND & $\hat{\mathrm{N}}=40,595+5,433^{* * *} \mathrm{I}_{\mathrm{N}}$ & 0,72 \\
\hline \multirow[t]{2}{*}{$\mathrm{P}$} & DRIS & $\hat{\mathrm{P}}=2,969+0,519^{* * *} \mathrm{IP}_{\mathrm{P}}$ & 0,53 \\
\hline & CND & $\hat{\mathrm{P}}=2,969+0,279^{* * *} \mathrm{I}_{\mathrm{P}}$ & 0,49 \\
\hline \multirow[t]{2}{*}{$\mathrm{K}$} & DRIS & $\hat{\mathrm{K}}=23,141+4,622^{* * *} \mathrm{IK}_{\mathrm{K}}$ & 0,67 \\
\hline & CND & $\hat{\mathrm{K}}=23,141+3,086^{* * *} \mathrm{I}_{\mathrm{K}}$ & 0,67 \\
\hline \multirow[t]{2}{*}{$\mathrm{Ca}$} & DRIS & $\mathrm{Câ}=11,597+4,233^{* * *} \mathrm{I}_{\mathrm{Ca}}$ & 0,74 \\
\hline & CND & $\mathrm{Ca}=11,597+2,368^{* * *} \mathrm{I}_{\mathrm{Ca}}$ & 0,78 \\
\hline \multirow[t]{2}{*}{$\mathrm{Mg}$} & DRIS & $\mathrm{Mg}=3,195+1,155^{* * *} \mathrm{I}_{\mathrm{Mg}}$ & 0,84 \\
\hline & CND & $\mathrm{Mg}=3,195+0,901^{* * *} \mathrm{I}_{\mathrm{Mg}}$ & 0,87 \\
\hline \multirow[t]{2}{*}{$\mathrm{S}$} & DRIS & $\hat{\mathrm{S}}=2,310+0,714^{* * *} \mathrm{I}_{\mathrm{S}}$ & 0,76 \\
\hline & CND & $\hat{\mathrm{S}}=2,310+0,529^{* * *} \mathrm{IS}_{\mathrm{S}}$ & 0,79 \\
\hline \multirow[t]{2}{*}{$\mathrm{B}$} & DRIS & $\hat{\mathrm{B}}=42,669+9,268^{* * *} \mathrm{I}_{\mathrm{B}}$ & 0,80 \\
\hline & CND & $\hat{\mathrm{B}}=42,669+7,022^{* * *} \mathrm{I}_{\mathrm{B}}$ & 0,80 \\
\hline \multirow[t]{2}{*}{$\mathrm{Cu}$} & DRIS & $\mathrm{Cu}=9,374+3,487 * * * \mathrm{I}_{\mathrm{Cu}}$ & 0,81 \\
\hline & CND & $\mathrm{Cu}=9,374+2,996^{* * *} \mathrm{I}_{\mathrm{Cu}}$ & 0,83 \\
\hline \multirow[t]{2}{*}{$\mathrm{Fe}$} & DRIS & $\mathrm{Fe}=90,072+26,525^{* * *} \mathrm{I}_{\mathrm{Fe}}$ & 0,75 \\
\hline & CND & $\mathrm{Fê}=90,072+21,633^{* * *} \mathrm{I}_{\mathrm{Fe}}$ & 0,76 \\
\hline \multirow[t]{2}{*}{$\mathrm{Mn}$} & DRIS & $\mathrm{Mn}=57,162+23,327 * * * \mathrm{I}_{\mathrm{Mn}}$ & 0,82 \\
\hline & CND & $\mathrm{Mn}=57,162+18,625^{* * *} \mathrm{I}_{\mathrm{Mn}}$ & 0,85 \\
\hline \multirow[t]{2}{*}{$\mathrm{Zn}$} & DRIS & $\mathrm{Z} \hat{\mathrm{n}}=56,756+24,213^{* * *} \mathrm{I}_{\mathrm{Zn}}$ & 0,86 \\
\hline & CND & $\mathrm{Zn}=56,756+19,688^{* * *} \mathrm{I}_{\mathrm{Zn}}$ & 0,89 \\
\hline
\end{tabular}

(1) Calculado conforme Alvarez V. \& Leite (1999), utilizando um fator de ajuste c = 1, Wadt et al. (1998a). ${ }^{(2)}$ Calculado conforme Khiari et al. $(2001 \mathrm{a}, \mathrm{b})$ porém com média geométrica dos constituintes da massa seca expressa em $\mathrm{mg} \mathrm{kg}^{-1}{ }^{(3)}>$ média $+0,5 \mathrm{~s}$. $* * *$ indica $\mathrm{p}<0,001$.

subpopulação de alta produtividade (Quadro 3), conforme observado por Kurihara (2004). A faixa ótima do teor de nutrientes, estimada como os teores correspondentes aos valores do índice zero $\pm 2 / 3$ desviopadrão, conforme Kurihara (2004), foi semelhante à faixa ótima estimada pelo DRIS (Quadro 3). Esse resultado é concordante com a elevada correlação observada por Khiari et al. (2001a) entre índices DRIS e CND ( $r=0,95$ a 0,99).

Os teores ótimos estimados para os nutrientes $\mathrm{N}$, $\mathrm{P}, \mathrm{K}, \mathrm{Ca}, \mathrm{Mg}, \mathrm{S}, \mathrm{B}, \mathrm{Cu}, \mathrm{Fe}, \mathrm{Mn}$ e Zn pelos métodos DRIS e CND foram idênticos ao teor médio na população de alta produtividade (Quadro 3), confirmando a distribuição normal das relações duais na população de alta produtividade, uma vez que, nesta condição, a média aritmética é igual à mediana.
$\mathrm{O}$ fato de o limite superior da faixa ótima de $\mathrm{N}$ estimada pelos métodos Chance Matemática, DRIS e CND substituir o limite inferior da faixa de suficiência proposta na literatura (Embrapa, 2003), de acordo com Kurihara (2004), deve-se ao fato de esta ter sido estabelecida nas décadas de 1970 e 1980 , quando a nutrição nitrogenada era dependente da aplicação de fertilizantes, diferentemente das condições atuais, em que o N é suprido às plantas de soja principalmente pela fixação biológica, podendo indicar maior eficiência de utilização desse nutriente atualmente.

A estimativa de faixas ótimas para teores de nutrientes pelos métodos Chance Matemática, DRIS e CND, com menor amplitude em relação às faixas estabelecidas na literatura (Embrapa, 2003), sugere, assim como para Kurihara (2004), a maior adequação do uso desses valores de referência, obtidos 


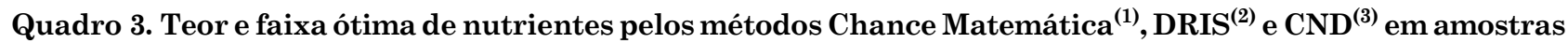
de soja coletadas na região sul do Estado de Mato Grosso do Sul, nos anos agrícolas de 2000/2001 e 2001/ 2002, em subpopulação de alta produtividade ${ }^{(4)}$. Dados citados na literatura ${ }^{(5)}$ foram incluídos para simples comparação

\begin{tabular}{|c|c|c|c|}
\hline Variável & Método & Faixa ótima & Teor ótimo \\
\hline \multirow[b]{2}{*}{$\mathrm{N}$} & & 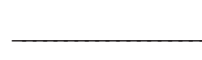 & - \\
\hline & $\begin{array}{l}\text { Chance Matemática } \\
\text { DRIS } \\
\text { CND } \\
\text { Literatura }\end{array}$ & $\begin{array}{l}36,0 \text { a } 47,8 \\
37,0 \text { a } 44,2 \\
37,0 \text { a } 44,2 \\
45,1 \text { a } 55,0\end{array}$ & $\begin{array}{l}40,6 \\
40,6 \\
40,6 \\
\text { na }\end{array}$ \\
\hline $\mathrm{P}$ & $\begin{array}{l}\text { Chance Matemática } \\
\text { DRIS } \\
\text { CND } \\
\text { Literatura }\end{array}$ & $\begin{array}{l}2,6 \text { a } 3,6 \\
2,8 \text { a } 3,2 \\
2,8 \text { a } 3,2 \\
2,6 \text { a } 5,0\end{array}$ & $\begin{array}{c}3,0 \\
3,0 \\
3,0 \\
\text { na }\end{array}$ \\
\hline $\mathrm{K}$ & $\begin{array}{l}\text { Chance Matemática } \\
\text { DRIS } \\
\text { CND } \\
\text { Literatura }\end{array}$ & $\begin{array}{l}16,6 \text { a } 25,5 \\
21,1 \text { a } 25,2 \\
21,1 \text { a } 25,2 \\
17,1 \text { a } 25,0\end{array}$ & $\begin{array}{c}23,0 \\
23,1 \\
23,1 \\
\text { na }\end{array}$ \\
\hline $\mathrm{Ca}$ & $\begin{array}{l}\text { Chance Matemática } \\
\text { DRIS } \\
\text { CND } \\
\text { Literatura }\end{array}$ & $\begin{array}{r}8,6 \text { a } 16,6 \\
10,1 \text { a } 13,1 \\
10,0 \text { a } 13,2 \\
3,6 \text { a } 20,0\end{array}$ & $\begin{array}{l}11,0 \\
11,6 \\
11,6 \\
\text { na }\end{array}$ \\
\hline $\mathrm{Mg}$ & $\begin{array}{l}\text { Chance Matemática } \\
\text { DRIS } \\
\text { CND } \\
\text { Literatura }\end{array}$ & $\begin{array}{l}1,7 \text { a } 4,3 \\
2,6 \text { a } 3,8 \\
2,6 \text { a } 3,8 \\
2,6 \text { a } 10,0\end{array}$ & $\begin{array}{c}3,2 \\
3,2 \\
3,2 \\
\text { na }\end{array}$ \\
\hline $\mathrm{S}$ & $\begin{array}{l}\text { Chance Matemática } \\
\text { DRIS } \\
\text { CND } \\
\text { Literatura }\end{array}$ & $\begin{array}{l}1,7 \text { a } 2,5 \\
2,0 \text { a } 2,7 \\
2,0 \text { a } 2,7 \\
2,1 \text { a } 4,0\end{array}$ & $\begin{array}{c}2,1 \\
2,3 \\
2,3 \\
\text { na }\end{array}$ \\
\hline B & $\begin{array}{l}\text { Chance Matemática } \\
\text { DRIS } \\
\text { CND } \\
\text { Literatura }\end{array}$ & $\begin{array}{l}35,1 \text { a } 57,3 \\
38,0 \text { a } 47,4 \\
38,0 \text { a } 47,4 \\
21,0 \text { a } 55,0\end{array}$ & $\begin{array}{l}42,0 \\
42,7 \\
42,7 \\
\text { na }\end{array}$ \\
\hline $\mathrm{Cu}$ & $\begin{array}{l}\text { Chance Matemática } \\
\text { DRIS } \\
\text { CND } \\
\text { Literatura }\end{array}$ & $\begin{array}{l}7,1 \text { a } 9,9 \\
7,4 \text { a } 11,3 \\
7,4 \text { a } 11,4 \\
6,0 \text { a } 14,0\end{array}$ & $\begin{array}{r}8,0 \\
9,4 \\
9,4 \\
\text { na }\end{array}$ \\
\hline $\mathrm{Fe}$ & $\begin{array}{l}\text { Chance Matemática } \\
\text { DRIS } \\
\text { CND } \\
\text { Literatura }\end{array}$ & $\begin{array}{l}52,0 \text { a } 135,9 \\
75,7 \text { a } 104,4 \\
75,6 \text { a } 104,5 \\
51,0 \text { a } 350,0\end{array}$ & $\begin{array}{l}84,0 \\
90,1 \\
90,1 \\
\text { na }\end{array}$ \\
\hline $\mathrm{Mn}$ & $\begin{array}{l}\text { Chance Matemática } \\
\text { DRIS } \\
\text { CND } \\
\text { Literatura }\end{array}$ & $\begin{array}{l}34,2 \text { a } 79,4 \\
45,0 \text { a } 69,4 \\
44,7 \text { a } 69,6 \\
21,0 \text { a } 100,0\end{array}$ & $\begin{array}{l}54,0 \\
57,2 \\
57,2 \\
\text { na }\end{array}$ \\
\hline $\mathrm{Zn}$ & $\begin{array}{l}\text { Chance Matemática } \\
\text { DRIS } \\
\text { CND } \\
\text { Literatura }\end{array}$ & $\begin{array}{l}21,0 \text { a } 82,2 \\
43,8 \text { a } 72,5 \\
43,6 \text { a } 69,9 \\
21,0 \text { a } 50,0\end{array}$ & $\begin{array}{c}44,3 \\
56,8 \\
56,8 \\
\text { na }\end{array}$ \\
\hline
\end{tabular}

Teor e faixa ótima estimados a partir da: ${ }^{(1)}$ mediana e os limites inferior e superior das classes de freqüência com maiores valores de chance matemática (Wadt, 1996), respectivamente. ${ }^{(2)}$ para um índice DRIS (calculado conforme Alvarez V. \& Leite (1999), utilizando um fator de ajuste $\mathrm{c}=1$, conforme Wadt et al. (1998a) igual a zero $\pm 2 / 3 \mathrm{~s}$, conforme Kurihara (2004), respectivamente. (3) para um índice CND (calculado conforme Khiari et al. (2001a,b), porém com média geométrica dos componentes da massa seca (expressa em $\mathrm{mg} \mathrm{kg}^{-1}$ ) igual a zero e $\pm 2 / 3 \mathrm{~s}$, conforme Kurihara $(2004)$, respectivamente. ${ }^{(4)}>$ média $+0,5 \mathrm{~s}$, com $\mathrm{n}=39$ talhões.

(5) Faixa de suficiência, conforme Embrapa (2003). na não avaliado. 
Quadro 4. Valores mínimos, máximos, médios e desvios-padrão (s) para teores de nutrientes em folhas de soja e produtividade, em amostras coletadas na região sul do Estado de Mato Grosso do Sul, nos anos agrícolas de 2000/2001 e 2001/2002, em subpopulações de alta e baixa produtividade ${ }^{(1)}$

\begin{tabular}{|c|c|c|c|c|c|c|c|c|}
\hline & \multicolumn{4}{|c|}{ Alta produtividade } & \multicolumn{4}{|c|}{ Baixa produtividade } \\
\hline & Mínimo & Máximo & Média & $\mathbf{s}$ & Mínimo & Máximo & Média & $\mathbf{s}$ \\
\hline Nitrogênio & 29,1 & 56,7 & 40,6 & 6,4 & 27,0 & 53,9 & 39,0 & 6,7 \\
\hline Fósforo & 2,2 & 3,7 & 3,0 & 0,4 & 1,3 & 4,2 & 2,7 & 0,6 \\
\hline Potássio & 13,0 & 29,0 & 23,1 & 3,8 & 12,0 & 34,5 & 22,1 & 4,5 \\
\hline Cálcio & 7,8 & 18,0 & 11,6 & 2,7 & 4,4 & 17,8 & 10,7 & 2,3 \\
\hline Magnésio & 1,8 & 5,0 & 3,2 & 1,0 & 1,7 & 6,0 & 3,6 & 1,0 \\
\hline Enxofre & 1,3 & 4,3 & 2,3 & 0,6 & 1,4 & 3,5 & 2,4 & 0,4 \\
\hline Boro & 26,9 & 61,0 & 42,7 & 7,9 & 23,8 & 59,7 & 42,6 & 7,3 \\
\hline Cobre & 2,6 & 17,2 & 9,4 & 3,3 & 4,2 & 15,3 & 9,6 & 3,3 \\
\hline Ferro & 32,0 & 164,9 & 90,1 & 24,8 & 30,9 & 240,8 & 78,9 & 31,2 \\
\hline Manganês & 19,3 & 122,9 & 57,2 & 20,2 & 19,0 & 170,0 & 68,4 & 30,7 \\
\hline Zinco & 29,0 & 122,0 & 56,8 & 20,9 & 21,0 & 174,0 & 49,9 & 24,0 \\
\hline Produtividade & 4.410 & 5.583 & 4.701 & 290 & 2.363 & 4.393 & 3.750 & 499 \\
\hline
\end{tabular}

$\overline{(1)}>$ e $\leq$ que média + 0,5 desvio-padrão, com número de talhões de 39 e 72 para as subpopulações de alta e baixa produtividade, respectivamente.

regionalmente, do que os valores estabelecidos em condições de solo, clima e potencial produtivo diferentes dos aqui considerados. Entretanto, deve-se ressaltar que os teores e as faixas ótimas estimados neste trabalho referem-se a um potencial produtivo elevado, uma vez que se consideraram talhões de alta produtividade aqueles que apresentaram produtividade superior a $4.400 \mathrm{~kg} \mathrm{ha}^{-1}$. Em trabalho semelhante, realizado a partir de amostras de soja coletadas nos Estados de Mato Grosso do Sul, Mato Grosso e Goiás, Kurihara (2004) verificou que os teores ótimos de Ca, $\mathrm{S}, \mathrm{Mn}$ e Zn em soja permanecem inalterados em produtividades inferiores a $3.600 \mathrm{~kg} \mathrm{ha}^{-1}$ e aumentam a partir desse potencial produtivo, enquanto os teores ótimos de $\mathrm{N}, \mathrm{P}, \mathrm{K}, \mathrm{Mg}, \mathrm{B}, \mathrm{Cu}$ e Fe não variam mesmo para produtividades superiores a $4.800 \mathrm{~kg} \mathrm{ha}^{-1}$.

Estudos são necessários para verificar a adequação da adoção de um critério único para determinação da amplitude da faixa representativa de uma condição de equilíbrio para todos os nutrientes avaliados, conforme realizado neste trabalho e por Kurihara (2004) para os métodos DRIS e CND, ou para definição de faixas de equilíbrio com amplitudes variáveis entre nutrientes, conforme sugerido por Savoy Júnior \& Robinson (1990) e Khiari et al. (2001a,b), a fim de melhor definir níveis críticos para a cultura da soja na região sul do Estado de Mato Grosso do Sul.

\section{CONCLUSÕES}

1. Os teores ótimos de nutrientes estimados pelos métodos DRIS e CND são idênticos ou muito próximos do teor médio na população de referência, o que não ocorre, de maneira geral, para o método Chance Matemática.

2. Os métodos Chance Matemática, DRIS e CND mostram-se promissores para a calibração de teores ótimos para a cultura da soja, a partir de dados provenientes de monitoramento nutricional de lavouras comerciais.

\section{LITERATURA CITADA}

ALVAREZ V., V.H. \& LEITE, R.A. Fundamentos estatísticos das fórmulas usadas para cálculo dos índices DRIS. B.Inf. Soc. Bras. Ci. Solo, 24:20-25, 1999.

COSTA, J.A. \& MARCHEZAN, E. Características dos estádios de desenvolvimento da soja. Campinas, Fundação Cargill, 1982. 30p.

DARA, S. T.; FIXEN, P.E. \& GELDERMAN, R.H. Sufficiency level and diagnosis and recommendation integrated system approaches for evaluating the nitrogen status of corn. Agron. J., 84:1006-1010, 1992.

EMPRESA BRASILEIRA DE PESQUISA AGROPECUÁRIA EMBRAPA. Tecnologias de Produção de soja - Região Central do Brasil 2004. Londrina, Embrapa Soja, 2003. 237p. (Sistemas de Produção, 4)

HARTZ, T.K.; MIYAO, E.M. \& VALENCIA, J.G. DRIS evaluation of the nutritional status of processing tomato. Hortic. Sci., 33:830-832, 1998. 
JONES, C.A. Proposed modifications of the diagnosis and recommendations integrated system (DRIS) for interpreting plant analyses. Comm.Soil Sci.Plant Anal., 12:785-794, 1981.

KHIARI, L.; PARENT, L.E. \& TREMBLAY, N. Critical compositional nutrient indexes for sweet corn at early growth stage. Agron. J., 93:809-814, 2001a.

KHIARI, L.; PARENT, L.E. \& TREMBLAY, N. The phosphorus compositional nutrient diagnosis range for potato. Agron. J., 93:815-819, 2001b.

KURIHARA, C.H. Demanda de nutrientes pela soja e diagnose de seu estado nutricional. Viçosa, MG, Universidade Federal de Viçosa, 2004. 101p. (Tese de Doutorado)

MAEDA, S. Interpretação do estado nutricional de soja pelo DRIS no Mato Grosso do Sul. Curitiba, Universidade Federal do Paraná, 2002. 107p. (Tese de Doutorado)

MALAVOLTA E.; VITTI, G.C. \& OLIVEIRA, S.A. Princípios, métodos e técnicas de avaliação do estado nutricional. In: MALAVOLTA, E.; VITTI, G.C. \& OLIVEIRA, S.A., eds. Avaliação do estado nutricional de plantas: princípios e aplicações. 2.ed. Piracicaba, Potafos, 1997. p.115-230.

REIS Jr., R.A. \& MONNERAT, P.H. DRIS norms validation for sugarcane crop. Pesq. Agropec. Bras., 38:379-385, 2003.

REIS Jr., R.A.; CORRÊA, J.B.; CARVALHO, J.G. \& GUIMARÃES, P.T.G. Diagnose nutricional de cafeeiros da região sul de Minas Gerais: normas DRIS e teores foliares adequados. R. Bras. Ci. Solo, 26:801-808, 2002.
RITCHIE, S.W.; HANWAY, J.J.; THOMPSON, H.E. \& BENSON, G.O. Como a planta de soja se desenvolve. Traduzido do original: Hoe a soybean plant develops. Special Report n.53 (Reprinted June, 1997). Ames,Iowa State University of Science and Technology Cooperative Extension Service, 1997.(POTAFOS. Arquivo do Agronômico, 11)

SAVOY Jr., H.J. \& ROBINSON, D.L. Norm range size effects in calculating diagnosis and recommendation integrated system indices. Agron. J., 82:592-596, 1990.

SFREDO, G.J.; LANTMANN, A.F.; CAMPO, R.J. \& BORKERT, C.M. Soja: nutrição mineral, adubação e calagem. Londrina, Embrapa/CNPSo, 1986. 51p. (Documentos, 17)

SILVA, G.G.C. Diagnose nutricional do eucalipto pelo DRIS, M-DRIS e CND. Viçosa, MG, Universidade Federal de Viçosa, 2001. 132p. (Tese de Mestrado)

WADT, P.G.S. Os métodos da chance matemática e do Sistema Integrado de Diagnose e Recomendação (DRIS) na avaliação nutricional de plantios de eucalipto. Viçosa, MG, Universidade Federal de Viçosa, 1996. 123p.( Tese de Doutorado)

WADT, P.G.S.; NOVAIS , R.F.; ALVAREZ V., V.H.; FONSECA, S. \& BARROS, N.F. Valores de referência para macronutrientes em eucalipto obtidos pelos métodos DRIS e Chance Matemática. R. Bras. Ci. Solo, 22:685-692, 1998b.

WADT, P.G.S.; NOVAIS, R.F; ALVAREZ V., V.H.; FONSECA, S.; BARROS, N.F. \& DIAS, L.E. Três métodos de cálculo de DRIS para avaliar o potencial de resposta à adubação de árvores de eucalipto. R. Bras. Ci. Solo, 22:661-666, 1998a. 